\title{
Fundamentos para processos gerenciais na prática do cuidado*
}

\author{
FUNDAMENTS FOR MANAGING PROCESS IN CARE PRACTICES
}

FUNDAMENTO PARA EL PROCESO GENERENCIAL EN LAS PRÁCTICAS DEL CUIDADO

\author{
Flavia Raquel Rossi', Maria Alice Dias da Silva Lima²
}

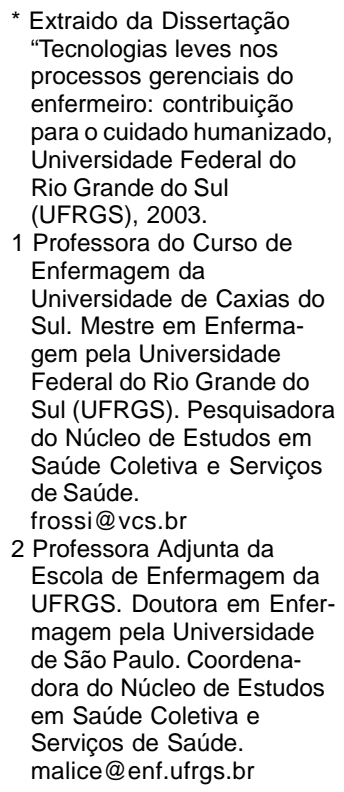

\begin{abstract}
RESUMO
Apresenta-se uma reflexão sobre elementos que estruturam o processo de trabalho em saúde e o agir do enfermeiro. Abordam-se o processo de trabalho em saúde, as tecnologias em saúde e o gerenciamento do cuidado como construtos que fundamentam a reflexão, com o objetivo de auxiliar na ampliação da visibilidade dos atos do enfermeiro. Ressalta-se a importância da utilização desses fundamentos para o exercício de práticas do cuidado nos processos gerenciais.
\end{abstract}

\section{DESCRITORES}

Cuidados de enfermagem (organização e administração). Papel do profissional de enfermagem.

Prestação de cuidados de saúde. Relações profissional-paciente.

\begin{abstract}
A reflection on the elements that structure the process of the nurse's work with health and his/ her action. The study deals with health work, technologies in health and the management of care as pillars that serve as bases for the reflection, with the aim of helping the enlargement of the visibility of the nurse's acts. The importance of those fundaments for the exercise of care practices in the managing processes is highlighted.
\end{abstract}

\section{KEY WORDS}

Nursing care (organization and administration).

Nurse's role.

Delivery of health care.

Professional and patient relations.

\section{RESUMEN}

El presente trabajo constituye una reflexión sobre los elementos que estructuran el proceso del trabajo en salud y el actuar del enfermero. Se aborda el proceso del trabajo en salud, las tecnologías en salud y el gerenciamiento del cuidado como constructores que fundamentan la reflexión, con el objetivo de ayudar a la ampliación de la visibilidad de los actos del enfermero. Se resalta la importancia de la utilización de estos fundamentos en el ejercicio de las prácticas del cuidado en los procesos gerenciales.

\section{DESCRIPTORES}

Atención de enfermería (organización y administración). Rol de la enfermera.

Prestación de atención de salud. Relaciones profesionalpaciente. 


\section{INTRODUÇÃO}

O contexto atual, caracterizado por rápidas mudanças, está exigindo dos indivíduos, das organizações e estabelecimentos, respostas diferenciadas no que diz respeito à efetividade dos serviços prestados. $\mathrm{Na}$ área da saúde, mais especificamente no âmbito da enfermagem, essa efetividade parece estar associada, não só a macro resultados sociais, econômicos e políticos, como também às questões do micro espaço pertinente ao cotidiano. Esse espaço, no qual ocorre a relação do enfermeiro com o usuário e com outros profissionais existentes no cenário da saúde, constitui e imprime determinadas características no processo de trabalho da enfermagem.

O enfermeiro atua além da relação individual com o usuário, deixando transparecer o caráter coletivo e de responsabilização também pelas atividades dos componentes da equipe de enfermagem ${ }^{(1-3)}$. Na maioria dos cenários de saúde brasileiros, os enfermeiros fazem a gerência da assistência ou do cuidado.

\section{Consideramos cuidado}

como um ato individual que prestamos a nós mesmos, desde que adquirimos autonomia mas é, igualmente, um ato de reciprocidade que somos levados a prestar a toda a pessoa que, temporariamente ou definitivamente tem necessidade de ajuda $^{(4)}$.

No âmbito da reciprocidade aparece o cuidado profissional - o cuidado de enfermagem, que necessita cada vez mais situar-se no contexto da vida, que lhe dá seu real significado. Mais especificamente deve situar-se no processo de vida e morte diante do qual os grupos humanos são colocados a cada dia, durante toda a sua existência. Para a sustentação desse contexto de vida é necessário considerar duas formas de cuidado: os cuidados habituais e cotidianos relacionados à manutenção e desenvolvimento da vida e os cuidados de reparação ou tratamento da doença ${ }^{(4)}$. Salientamos que esses últimos impregnaram de tal forma os ambientes próprios ao cuidado em função do modelo biomédico de assistência que, embora com algumas flexibilizações, ainda é realizado isolando o indivíduo do seu meio, do seu grupo de inserção e até mesmo de si próprio.

A atenção a esses aspectos é de fundamental importância ao ser considerado o gerenciamento do cuidado, tendo em vista que os dois tipos de cuidado não se excluem mutuamente. Ambos devem ser foco das ações do enfermeiro e constantemente considerados diante situações que exigem prestação de cuidados. Para dar conta desse cuidado o enfermeiro deve inserir-se nos espaços que lhe dizem respeito, quer seja junto ao usuário ou às equipes de saúde, de forma consciente e direcionada às necessidades especificas dos sujeitos para que sejam cuidados em sua totalidade e não apenas em suas partes ${ }^{(5)}$. A atenção direcionada à necessidade do indivíduo (cuidador ou ser cuidado) pode ser um determinante para a humanização do cuidado, considerada como relação dialógica entre homens que proporciona o desenvolvimento de cada um, na qual a individualidade, as crenças, as características pessoais, a linguagem, entre outras coisas, são $\operatorname{respeitadas}^{(5-6)}$.

Refletindo sobre esse contexto, questionamos: como podemos falar em cuidado humanizado se perpetuamos a idéia de redução dos sujeitos humanos, quer sejam produtores ou receptores do cuidado, à condição de instrumentos dos estabelecimentos e das organizações, transformados em recursos ou objetos? Podemos afirmar que produzimos cuidado humanizado, sendo que se percebe ainda em muitas realidades que os próprios trabalhadores se vêem desprovidos dessa dimensão humanizada? Como podemos garantir um cuidado humanizado ao usuário se o próprio cuidador não se vê e não se sente respeitado nessa dimensão?

Nesse aspecto, cabe ressaltar que o desafio está em governar para produzir sujeitos, o que implica em deixar que a sensibilidade, o desejo e as necessidades dos mesmos penetrem em todos os poros da organização de forma que todos venham a sentir respeito, autonomia, justiça, ética e liberdade ${ }^{(7)}$ !

É possível que a fonte do cuidado humanizado esteja no engajamento do enfermeiro em trabalhar propostas que contenham essa concepção, sendo necessário para isso, que ela seja reconhecida como possível pelos profissionais e que esses utilizem tecnologias diferenciadas capazes de aproximar os indivíduos e conduzi-los à busca da satisfação das necessidades existentes ${ }^{(5)}$.

As questões expostas introduzem à necessidade de revisão de conceitos relacionados às questões gerenciais em enfermagem, com o intuito de estimular reflexões e desafios para novas possibilidades do "fazer", tendo em vista o indivíduo, como centralidade dos processos gerenciais do enfermeiro.

Para o entendimento e contextualização desses processos gerenciais torna-se necessária uma fundamentação teórica que, além de ampliar a visibilidade do tema, permita discussões de aspectos essenciais para a ação do enfermeiro e sua associação com o cuidado humanizado. Dessa forma, os pro-
Fundamentos para processos gerenciais nas práticas do cuidado 
Flavia Raquel Rossi Maria Alice Dias da Silva cessos de trabalho em saúde, as tecnologias em saúde e o gerenciamento do cuidado serão a bordados como os pilares fundamentais desta contextualização.

\section{PROCESSO DE TRABALHO EM SAÚDE}

Ao abordar os aspectos relativos aos processos gerenciais do enfermeiro são oportunas algumas considerações relativas ao trabalho em saúde, analisando espaços do cotidiano, nos quais ocorrem as relações do enfermeiro com o usuário e com os profissionais da equipe de saúde.

O trabalho é entendido como uma ação cotidiana, que coloca homens em relação com outros homens e com a natureza, dentro de um determinado processo social e histórico ${ }^{(8)}$, sendo considerado também como uma atividade genérico-social quando é útil para os indivíduos, cumpre uma função social e leva em conta a satisfação das necessidades sociais em tempo socialmente necessário para produzi-lo ${ }^{(2)}$.

O trabalho em saúde é considerado como sendo da esfera não material, que se completa no ato de sua realização. Portanto, a objetividade e a subjetividade lhe são inerentes, tendo em vista que o objeto que o constitui são seres humanos e consequientemente as intervenções técnicas serão sempre permeados por relações interpessoais ${ }^{(2,9)}$.

É um trabalho em serviços e como tal atende a dois aspectos. O primeiro é o de preservar, respeitar e reconhecer a particularidade, a individualidade, a variabilidade das situações e necessidades dos usuários e o segundo é o de estar de acordo com certas regras, regulamentos e valores gerais. No entanto, somente será bem sucedido se produzir o equilíbrio entre $\operatorname{ambos}^{(2)}$.

O processo de trabalho é um jogo entre produção, consumo e necessidades dos indivíduos e, na área da saúde, de forma muito particular, produz atos de saúde que perseguem a produção do cuidado. Os processos de trabalho, embora tenham bases mecanicistas, possuem resultados que dependem das relações entre as pessoas e que decorrem da interação de dois componentes, denominados: trabalho vivo e trabalho morto ${ }^{(10)}$.

O trabalho vivo situa-se na dimensão do cotidiano e constitui-se das ações propriamente ditas executadas pelos trabalhadores de saúde na sua relação com o usuário e diríamos também, entre os próprios trabalhadores. Faz parte do trabalho que é instituinte, que estabelece, que cria, que está em processo e em ação. Na dimensão do trabalho vivo são operacionalizadas as noções de autonomia e auto-governo dos indivíduos. Há a preservação do espaço de julgamento, de decisão, de processo reflexivo, de integração, de intencionalidade, de utilização de saberes específicos para situações em particular que se apresentam na atenção ao usuário ${ }^{(2,10)}$.

O trabalho morto é resultado de um trabalho humano anterior. É composto por todos os elementos que estão envolvidos nos processos de trabalho, como ferramentas ou matéria-prima, assim como também por componentes vinculados ao saber e ao modo de atuar dos profissionais envolvidos no trabalho $^{(10)}$.

Entendemos que o processo de trabalho em saúde se constitui como um espaço de relação e de movimento constante entre o trabalho morto e o trabalho vivo, sendo que ambos são concomitantemente condicionantes e condicionados. O cuidado, por sua vez, enquanto parte desse processo e reconhecido como uma relação entre os indivíduos, concretiza-se no espaço do trabalho vivo.

Esse entendimento é fonte de preocupação quando associado à humanização do cuidado, pois nos espaços do trabalho vivo ainda estão presentes resquícios mecanicistas, fragmentados e bem estruturados demonstrando uma certa invasão, impregnação ou soberania do trabalho morto sobre o vivo.

Embora o trabalho vivo em ato seja quase que plenamente capturado por forças instituídas, ainda existe espaço para exercer variações por se tratar de um trabalho que acontece no exato momento em que ocorre a relação entre os indivíduos ${ }^{(10)}$ - usuário/trabalhador, trabalhador/trabalhador, no qual inevitavelmente aparece o campo da subjetividade humana.

No entanto, em algumas instâncias, a prática nos mostra um não aproveitamento por parte do enfermeiro, das ricas oportunidades de melhorias, resolutividade, crescimento pessoal e profissional existentes nas relações cotidianas, resultantes dos vários encontros feitos com o usuário e com a equipe. Os contatos, embora possam acontecer entre os mesmos indivíduos, se fazem em momentos e situações diferentes, sendo, portanto, sempre novos. O que parece estar fixo na ação profissional é a defasagem de atualização e contextualização dos diferentes momentos. Parece haver uma dificuldade, uma acomodação e uma certa passividade de alguns profissionais diante da necessidade de reciclar a sua prática, de buscar novas tecnologias, novas formas de fazer e principalmente novas formas de ser no trabalho. 
Esses posicionamentos são provenientes de uma mescla de fatores, incluindo a subjetividade dos profissionais, os resquícios da historicidade da profissão de enfermagem marcada entre tantas outras coisas pela subalternidade, e ainda outras que advêm de questões organizacionais e dos modelos assistenciais e administrativos existentes nos estabelecimentos de saúde.

Há que se levar em consideração que os profissionais podem reproduzir ou modificar os processos de trabalho e os modelos de assistência, dos quais emana o cuidado. Nesse sentido, é importante salientar que, determinados tipos de organizações têm como missão controlar o auto-governo dos indivíduos, com o receio de que haja uma alteração na lógica de seu funcionamento, assim como enfatiza-se que a construção de tecnologias para a ação do trabalho vivo em ato e para a gestão desse trabalho, abrem espaços interrogadores e de ruptura dos processos institucionalizados, transformando o processo de trabalho em lugar estratégico de mudança ${ }^{(10)}$.

Embora o enfermeiro seja um dos profissionais da equipe de saúde que vivencia a construção, as repercussões e resultados do trabalho vivo em ato em decorrência de sua inserção nos processos de trabalho, do seu potencial polivalente, mediador e articulador, ele nem sempre está apto, tem espaço e/ ou desejo de romper com os processos institucionalizados. Os aspectos concretos e estruturados dão segurança aos indivíduos, tendo em vista o receio e a resistência em se desestabilizarem e se lançarem rumo a novos caminhos ${ }^{(5)}$.

Diante disso, decorrem lacunas advindas do fato de o enfermeiro, ainda em muitas instâncias, não ser capaz de explorar com mais intensidade o trânsito e a representatividade que tem de uma ponta a outra do sistema de saúde (desde o cuidado ao usuário até os processos de gerenciamento e gestão), no sentido de atuar e investir na qualificação do trabalho vivo em ato, em encadear suas ações com as de outros profissionais, em utilizar e construir novas tecnologias, em incorporar de vez o papel fundamental que exerce na organização e estruturação dos processos de trabalho em saúde - cenário onde acontece o cuidado.

Essas reflexões nos reportam a um outro aspecto importante na determinação de processos de trabalho em saúde que é a construção coletiva. O enfermeiro tem papel preponderante nessa construção por ser um dos articuladores dos processos de trabalho interagindo com todos os profissionais da saúde, e coordenando a organização desses processos. Não é raro também que ele seja o ponto de convergência e distribuição de informações para o usuário, para a grande maioria dos profissionais da equipe de saúde, assim como para os diferentes serviços que fazem parte do universo hospitalar ${ }^{(11)}$.

É necessário pontuar, no entanto, a existência de um problema nos processos de trabalho coletivo: o coletivo está fragmentado e dessa forma fragmenta também o trabalho ${ }^{(12)}$. $\mathrm{O}$ trabalho coletivo resume-se, em muitas instâncias, na prática simplista de um conjunto de pessoas trabalhando no mesmo espaço. Os objetivos do trabalho ainda são regidos por pontos de vista, necessidades e interesses particularizados dos trabalhadores, a sua linguagem não é unificada, assim como os seus resultados aparecem fragmentados, ficando aquém do esperado para a concretização do verdadeiro trabalho coletivo que implica em participação, comunicação e continuidade de ações efetivas ${ }^{(5)}$.

Ao associar esses aspectos com o fato de que os processos de trabalho acontecem em uma rede que se alimenta reciprocamente, impulsionados pelas necessidades dos indivíduos ${ }^{(2)}$, valem algumas considerações. A primeira é relacionada às necessidades dos indivíduos, entendendo-as como carências pertinentes a eles, social e historicamente construídas e que só podem ser captadas e trabalhadas em sua dimensão individual ${ }^{(13)}$.

Quando se fala em trabalho em saúde, de quais necessidades falamos? Quais os indivíduos que são considerados em suas necessidades dentro dos processos de trabalho em saúde? Embora tenhamos claro que o usuário tem suas necessidades alinhadas ao objetivo final do processo de trabalho em saúde e que as necessidades dos trabalhadores também aparecem e interferem nas relações que definem os processos de trabalho e seus resultados, esses fatores não aparecem sedimentados de forma adequada dentro do trabalho coletivo, tendo em vista que as necessidades de alguns usuários e trabalhadores continuam não sendo satisfeitas de forma contínua e adequada, existindo inclusive equívocos de interpretação da satisfação dessas necessidades.

O segundo grupo de considerações relacionase com o produto esperado como resultado do trabalho em saúde. O produto esperado é o mesmo para todos os profissionais que participam do processo de trabalho em saúde? Este produto tem o mesmo significado para todos os profissionais que auxiliam na sua construção? Esse produto - que deveria ser o usuário recuperado e cuidado de forma humanizada - ainda não é comum a todos e a satisfação de suas necessidades ainda vem parcelada e alinhada com a satisfação dos profissionais que com eles atuam, assim como com o cumpri- 
Flavia Raquel Rossi

Maria Alice Dias da Silva mento de aspectos organizacionais, chegando em alguns momentos a ser antagônica ${ }^{(5)}$.

Diante dessas reflexões vale ressaltar que a reunificação do trabalho só poderá ser feita pela complementaridade consciente das atividades, ainda que cada trabalhador execute uma parte do trabalho total $^{(12)}$.

Para isso se faz necessária a transversalidade de determinadas ações dos trabalhadores de saúde, tais como as direcionadas à resolutividade, ao acolhimento, ao vínculo, entre outras, assim como a garantia do encadeamento entre as várias e diferentes relações entre trabalhador/usuário e trabalhador/trabalhador que ocorrem no cuidar, que são interdependentes em função de um produto que é comum e que caracterizam o trabalho coletivo.

\section{TECNOLOGIAS EM SAÚDE}

As diferentes possibilidades tecnológicas, assim como o desenvolvimento tecnológico devem ser contempladas constantemente no cotidiano dos estabelecimentos de saúde na busca de resultados mais efetivos das ações, evitando a prática de ações insuficientes para a satisfação dos usuários.

Através do desenvolvimento tecnológico acontece uma transição natural quanto à sua utilização e inserção nos ambientes de trabalho e, na área da saúde, essas transições têm imprimido alterações significativas nos processos de trabalho ao longo dos tempos. Essas alterações são evidenciadas através de características do parcelamento dos processos de trabalho, no perfil e na qualificação dos trabalhadores e nas variações do mercado de trabalho $^{(14)}$.

Temos como tecnologias envolvidas no trabalho em saúde:

Tecnologias leves (como no caso das tecnologias de relações do tipo produção de vínculo, autonomização, acolhimento, gestão como uma forma de governar processos de trabalho), leveduras (como no caso de saberes bem estruturados que operam no processo de trabalho em saúde, como a clínica médica, a clínica psicanalítica, a epidemiologia, o taylorismo, o fayolismo) e duras (como no caso de equipamentos tecnológicos do tipo máquinas, normas, estruturas organizacionais) $)^{(14)}$

As tecnologias leves podem ser importantes ferramentas de gerenciamento utilizadas pelo enfermeiro na busca da qualidade do cuidado prestado aos usuários, pois estabelecem momentos de interces- são entre trabalhadores e usuários e permitem a real possibilidade de reconhecimento e satisfação das necessidades dos indivíduos. Dessa forma, podem ser utilizadas para a concretização e sustentação de um modelo de assistência que venha a contemplar um cuidado humanizado.

É importante considerar que a reestruturação produtiva na área da saúde acompanha as interações que o modelo médico hegemônico vem sofrendo pelas mudanças operadas pela introdução de novos equipamentos. Essa entrada de equipamentos não anula momentos singulares do trabalho em saúde, insubstituíveis como as dimensões típicas da produção do ato cuidador e das interações gerenciais $^{(15)}$.

A entrada de equipamentos, denominada de tecnologia dura, nos processos produtivos em saúde, sob a modelagem de gestão médico-hegemônica não mais provoca reestruturação produtiva e nem compõe uma transição dos modelos produtivos e assistenciais. Ao contrário, a transição tecnológica busca atingir o núcleo tecnológico do trabalho vivo em ato, procurando novas conformações dos atos de saúde, descentrando o trabalho em saúde até mesmo dos equipamentos e dos especialistas ${ }^{(14)}$.

Nessa concepção situa-se a tecnologia leve, conceituada como:

\begin{abstract}
a tecnologia de (e das) relações - dos intercessores, no interior dos processos que podem gerar alterações significativas no modo de se trabalhar em saúde [....] sob uma ótica analisadora pautada pela ética do compromisso com a vida e expressa em ato nas dimensões assistenciais do trabalho vivo em saúde, como a relação de acolhimento, a criação do vínculo, a produção da resolutividade e a criação de maiores graus de autonomia, no modo das pessoas andarem na vida $^{(10)}$.
\end{abstract}

O uso das tecnologias leves contempla a existência de um objeto de trabalho dinâmico, em contínuo movimento, não mais estático, passivo ou reduzido a um corpo físico. Esse objeto exige dos profissionais da saúde, especialmente do enfermeiro, uma capacidade diferenciada no olhar a eles concedido a fim de que percebam essa dinamicidade e pluralidade, que desafiam os sujeitos à criatividade, à escuta, à flexibilidade e ao sensível ${ }^{(5)}$.

É importante enfatizar que os instrumentos de trabalho não são naturais, são construídos historicamente pelo sujeito que assim entende sua possibilidade de intervenções sobre o objeto. Se o objeto muda em suas necessidades, as intervenções do trabalhador também devem mudar ${ }^{(8)}$. 
Fica claro que não se pode restringir a tecnologia à dimensão constituída pelos instrumentos materiais de trabalho. A tecnologia refere-se aos nexos técnicos estabelecidos no interior do processo de trabalho entre a atividade operante e os objetos de trabalho através dos instrumentos utilizados para a sua concretização ${ }^{(16)}$. É importante incluir também o saber, entendido como a posse e a manipulação de objetos de trabalho no seio do processo de produção, como uma tecnologia que organiza a prática de saúde ${ }^{(8)}$.

Para abordar tecnologias em saúde alinhadas às necessidades atuais dos usuários e dos próprios serviços de saúde é necessário ir além da dimensão das máquinas e equipamentos, cujo impacto da sua utilização na área da saúde auxilia na garantia e manutenção da vida por todos os indivíduos, quer sejam usuários ou trabalhadores de saúde, porém não substitui e nem supera outras interações tecnológicas também vitais para a busca da inteireza dos indivíduos participantes do processo.

De forma complementar é importante enfatizar que a tecnologia engloba o processo de trabalho como um todo e tem conotações técnicas e sociais. Do ponto de vista técnico consiste na organização do complexo de recursos destinado ao cumprimento de determinada tarefa, ou seja, do ordenamento recebido pelos diversos instrumentos de trabalho (não só maquinarias), para o processamento dos materiais de trabalho ${ }^{(17)}$.

Do ponto de vista social, a incorporação de nova tecnologia redefine o trabalho em saúde afetando as relações internas da equipe de trabalho, assim como as relações dos profissionais com o usuário. Há a existência de uma tecnologia de organização, que se reporta principalmente aos recursos humanos que fazem parte dos serviços de saúde, considerando a noção de equipe e o tipo de pessoas que atuam na área assistencial ${ }^{(17)}$.

Embora exista o reconhecimento do forte componente de centralização na figura do médico nas práticas de saúde, ao fazer a observação das relações internas dessas práticas é constatada também a captura de determinadas tecnologias por outros profissionais. A enfermagem vivencia essa situação nas diferentes especialidades de atuação, assim como nas questões que privilegiam aspectos organizacionais. Nas equipes é evidenciada a relação direta dos diferentes status profissionais, mesmo que não explícitos, com a complexidade das tecnologias apreendidas e utilizadas. Como exemplo dessa realidade temos o grande interesse da maioria dos acadêmicos de enfermagem pela alta complexidade clínica e dos equipamentos existen- tes nos centros de terapia intensiva, assim como a auto-imagem e o reconhecimento elevados dos profissionais que atuam em intensivismo, também pelo domínio clínico e pelo manuseio de equipamentos sofisticados, como se fossem os únicos arsenais tecnológicos capazes de responderem às necessidades dos usuários.

Há prejuízo também nas relações enfermeiro/ usuário e enfermeiro/equipe, pois essas relações, além de serem intermediadas pelos equipamentos, também o são pela engrenagem organizacional normativa e estruturada.

É oportuno reforçar que é imprescindível o contato e a permeabilidade constante dos diferentes saberes utilizados pelos profissionais da saúde na sua prática específica, assim como os pertinentes à organização dos serviços de saúde, com o tecido social. Esse contato incentiva a utilização e a criação de tecnologias mais contextualizadas, que respondam de forma mais efetiva às necessidades integrais dos indivíduos inseridos nos processos de trabalho - usuários e trabalhadores, repercutindo nos próprios estabelecimentos, na organização da saúde e, conseqüentemente, na sociedade como um $\operatorname{todo}^{(5)}$.

Para tanto, é essencial a apreensão dessas tecnologias pelos diferentes profissionais de saúde, agregada à atividade gerencial. Nessa instância é inquestionável a atuação do enfermeiro para a construção desse caminho.

\section{GERENCIAMENTO DO CUIDADO}

As questões gerenciais têm suscitado muitas preocupações em todos os segmentos da sociedade, tendo em vista que delas depende a consecução dos objetivos organizacionais. $\mathrm{Na}$ área da saúde existe porém, uma particularidade: nela encontra-se, no interior dos objetivos esperados, o almejado resultado proveniente da trajetória do usuário através dos serviços de saúde e da atenção a ele prestada.

Ao enfocar os resultados da atenção ao usuário nos serviços de saúde é conveniente salientar que apesar da alta velocidade das mudanças do ambiente nas últimas décadas ter contribuído para a flexibilidade das empresas, para a procura de agilidade nas respostas às demandas do ambiente, para um lugar destacado ao capital humano e para uma gestão de produtos e serviços centrados no cliente, a vida das pessoas tem se alterado muito pouco ${ }^{(1)}$.

Em âmbito hospitalar verifica-se que, apesar da modernização e evolução em nível organizacional e
Fundamentos para processos gerenciais nas práticas do cuidado 
Flavia Raquel Rossi Maria Alice Dias da Silva tecno-científico, em grande parte desses estabelecimentos, nos níveis intermediários da hierarquia administrativa, nos quais situa-se freqüentemente o enfermeiro, as funções gerenciais são confusas, diluídas e um tanto distantes do usuário e das práticas interdisciplinares. Esse modo de ser da organização tem interferência constante nos processos de trabalho, no modelo de assistência e conseqüentemente na qualidade do cuidado prestado.

No entanto, o usuário de saúde ao pontuar a sua insatisfação com a atenção recebida, não a tem atrelado à falta de conhecimentos técnico-científicos, mas sim, à falta de interesse e responsabilização dos profissionais acerca de seus problemas e necessidades $^{(17)}$, deixando transparecer que há algo errado na forma como essa atenção está sendo conduzida.

É necessário dar ênfase ao fato de que

no campo da saúde o objeto não é a cura, ou promoção e proteção da saúde, mas a produção do cuidado, por meio do qual se crê que se poderá atingir a cura e a saúde, que são de fato os objetivos a que se quer chegar(14).

É impreterível, portanto, reconhecer o cuidado como foco possível e necessário de ser gerenciado dentro do universo organizacional em uma dimensão que extrapole o tecnicismo.

No entanto, o modelo clínico de assistência e o modelo científico de administração ainda embaçam o olhar do enfermeiro enquanto exerce o papel gerencial, tirando-lhe muitas vezes a clareza de quais ações fazem parte da concretização desse cuidado. O enfermeiro, na área hospitalar, ainda se perde no exercício das funções administrativas, voltado para a tecnoburocracia, fazendo o gerenciamento das unidades de internação com a lógica do gerenciamento científico, enfatizando o controle mecânico das atividades, muitas vezes desenvolvidas por outros agentes $^{(18)}$.

O diferencial que irá demarcar o gerenciamento feito pelos enfermeiros como sendo verdadeiramente gerenciamento do cuidado será o seu posicionamento diante do modo como desenvolvem o trabalho. É necessário levar em conta como esses profissionais se envolvem em suas atividades, que saberes utilizam e principalmente a quem ou ao que respondem quando os utilizam.

Fazer gerenciamento do cuidado implica em têlo como foco das ações profissionais e em utilizar os saberes administrativos como mais uma tecnologia (leve-dura) no sentido de sua concretização. No caso específico da enfermagem essa concre- tização pode ser através de ações diretas do profissional com o usuário, por intermédio de delegação e ou articulação com outros profissionais da equipe de saúde. O enfermeiro gerencia o cuidado quando o planeja, quando o delega ou o faz, quando prevê e provê recursos, capacita sua equipe, educa o usuário, interage com outros profissionais, ocupa espaços de articulação e negociação em nome da concretização e melhorias do cuidado(5).

É impreterível que o enfermeiro ocupe os espaços de discussão e negociações estratégicas para a garantia do cuidado e agregue diferentes tecnologias às suas ações, levando em consideração, de forma muito especial, o conteúdo subjetivo e sensível que permeia o mundo do cuidado. É importante considerar o teor significativo, pessoal, imprevisível, não rotineiro existente nas diversas relações entre os sujeitos que fazem parte dos processos de trabalho e que precisam ser reconhecidas para que os objetivos individuais - dos usuários e trabalhadores - e da própria organização sejam alcançados.

Para tanto, são necessários processos gerenciais que incorporem conhecimentos, atitudes e ações tanto da ordem do racional como do sensível, assim como o entrelaçamento e a aproximação entre o cuidar e o gerenciar. Essas aproximações fazem parte de um novo paradigma da enfermagem que está sendo construído na atualidade ${ }^{(18)}$.

Nesse contexto em construção é necessária especial atenção aos recursos humanos, pois todo o profissional de saúde, independente do papel que desempenhe

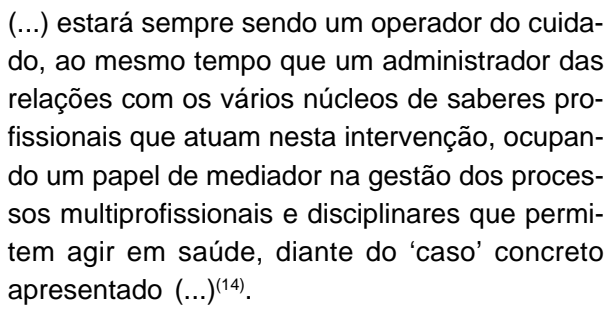

O enfermeiro pode reconhecer-se nessa citação, assim como pode reconhecer também os profissionais que com ele atuam e que, por sua vez, também cuidam. Ao ser apreendida essa concepção tornase difícil seguir com práticas calcadas nos modelos tradicionais de administração científica e burocrática embutidos no gerenciamento de enfermagem de até então, tendo em vista a possibilidade de abertura de espaços e a legitimação de possibilidades de um fazer diferente, que pode ser partilhado, co-responsabilizado e descentralizado.

Temos a partir dessa visão a inclusão do outro nos processos gerenciais, não como recurso, mas 
sim como sujeito das ações. Esse outro pode ser o colega de equipe, o profissional de outra área ou o próprio usuário, e vem dotado de saberes, emoções, limitações, potencialidades, poderes - enfim de características humanas, e define juntamente com o enfermeiro a qualidade do cuidar $^{(5)}$.

Dessas considerações emerge o fato de que o trabalho gerencial é coletivo,

cada vez mais interdependente, determinado pelas demandas e necessidades do cliente e que o seu sucesso está ancorado na solidariedade, na ética e em times de trabalho que respeitem e valorizem as diferenças, enquanto aspectos complementares de grupos heterogêneos de pessoas que colocam à disposição do cliente seus conhecimentos e habilidades ${ }^{(1)}$.

Sendo assim, devem ser considerados fatores como a capacidade e envolvimento dos gestores, dos profissionais que comandam em instâncias mais elevadas no interior dos estabelecimentos, dos profissionais das áreas de apoio, dos profissionais da área da assistência e o usuário como imprescindíveis para o sucesso das ações gerenciais focadas no cuidado.

Enfatizamos o envolvimento dos trabalhadores de saúde e usuários para que haja mudanças nas formas de se fazer gestão e assistência, pois são eles que refletem o cotidiano. Dessa forma, instrumentalizá-los e auxiliar na sua construção enquanto sujeitos que operam nesse cotidiano é imprescindível $^{(19)}$.

É necessário que o trabalho do enfermeiro, ao gerenciar o cuidado, resulte em mais do que simplesmente organizar o serviço segundo padrões de eficiência, mas acima de tudo, consiga também construir sujeitos sociais nesse território singular de prática, tendo em vista que são esses sujeitos que con- tribuem para a concretização e dão características ao cuidado $^{(20)}$.

\section{CONSIDERAÇÕES FINAIS}

É preciso, portanto, refletir criticamente e lançar novo olhar sobre os processos de gerenciamento do cuidado, a fim de que seja construída uma nova realidade organizacional alinhada a melhores práticas. Essas novas práticas do cuidado devem estar sustentadas pela utilização diferenciada e inovadora dos distintos tipos de tecnologias, que não só as pertencentes aos modelos tecnológicos utilizados no desenvolvimento da administração científica e no modelo clínico de assistência.

O gerenciamento do cuidado exige dos profissionais da saúde e particularmente do enfermeiro, uma visão que integre e acolha os valores e lógicas diferenciadas impressos nas necessidades dos usuários, não manifestos ou reconhecidos até algum tempo atrás. Diante dessa realidade é necessário um mergulho dos profissionais nos aspectos subjetivos das inter-relações, na sua potencialidade de criatividade e autonomia, no sentido de darem conta da satisfação das necessidades emergentes nos processos de trabalho, cujas soluções não são encontradas em nenhum manual de normas, rotinas e técnicas.

O grande compromisso e desafio de quem gerencia o cuidado hoje é o de valorizar e habilitarse para utilizar as relações como uma tecnologia, no sentido de edificar um cotidiano, por intermédio da construção mútua entre os sujeitos. E, através dessas mesmas relações, dar sustentação à satisfação das necessidades dos indivíduos e valorizar trabalhadores e usuários como potentes para intervirem no trabalho vivo em ato - espaço de concretização do cuidado.
Fundamentos para processos gerenciais nas práticas do cuidado

\section{REFERÊNCIAS}

(1) Alves M. A gerência do cuidado de enfermagem frente a novos modelos de gestão. In: Anais do $50^{\circ}$ Congresso Brasileiro de Enfermagem; 1998 set. 20-25; Salvador. Salvador: ABEn-Seção-BA; 1999. p. 153-8.

(2) Peduzzi M. Equipe multiprofissional de saúde: a interface entre trabalho e interação [dissertação]. Campinas (SP): Faculdade de Ciências Médicas da UNICAMP; 1998.

(3) Pires D. A estrutura objetiva do trabalho em saúde. In: Leopardi MT, organizadora. Processo de trabalho em saúde: organização e subjetividade. Florianópolis: Papa-Livros; 1999.
(4) Collière MF. Promover a vida: da prática das mulheres de virtude aos cuidados de enfermagem. Lisboa: Sindicato dos Enfermeiros Portugueses; 1989.

(5) Rossi FR. Tecnologias leves nos processos gerenciais do enfermeiro: contribuição para o cuidado humanizado [dissertação]. Porto Alegre (RS): Escola de Enfermagem da UFRGS; 2003.

(6) Freire P. Educação como prática da liberdade. $24^{\mathrm{a}}$ ed. Rio de Janeiro: Paz e Terra; 2000.

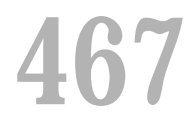

Rev Esc Enferm USP 2005; 39(4):460-8. 
Flavia Raquel Rossi Maria Alice Dias da Silva
(7) Campos GWS. Considerações sobre a arte e a ciência da mudança: revolução das coisas e reforma das pessoas. O caso da saúde. In: Cecílio LCO, organizador. Inventando a mudança na saúde. São Paulo: HUCITEC; 1997. p. 29-116.

(8) Gonçalves RBM. Práticas de saúde: processo de trabalho e necessidades. São Paulo: CEFOR; 1992. (Cadernos CEFOR. Textos; 1)

(9) Pires D. Reestruturação produtiva e trabalho em saúde no Brasil. São Paulo: Annablume; 1998.

(10) Merhy EE. Em busca do tempo perdido: a micropolítica do trabalho vivo em saúde. In: Merhy EE, Onocko R, organizadores. Agir em saúde. São Paulo: HUCITEC; 1997. p. 71-112.

(11) Rodrigues FCP, Lima MADS. A multiplicidade de atividades realizadas pelo enfermeiro em unidades de internação. Rev Gaúcha Enferm 2004; 25(3): 314-22.

(12) Vaz MRC. Trabalho em saúde: expressão viva da vida social. In: Leopardi MT, organizadora. Processo de trabalho em saúde: organização e subjetividade. Florianópolis: Papa-Livros; 1999. p. 57-70.

(13) Cecilio LCO. A necessidade de saúde como conceito estruturante na luta pela integralidade e eqüidade na atenção. In: Pinheiro R, Mattos RA, organizadores. O sentido da integralidade na atenção e no cuidado à saúde. Rio de Janeiro: ABRASCO; 2001. p. 113-26
(14) Merhy EE. Saúde: a cartografia do trabalho vivo. São Paulo: HUCITEC; 2002.

(15) Merhy EE. Em busca de ferramentas analisadoras das tecnologias em saúde: a informação e o dia a dia de um serviço, interrogando e gerindo trabalho em saúde. In: Merhy EE, Onocko R, organizadores. Agir em saúde. São Paulo: HUCITEC; 1997. p. 11350

(16) Gonçalves RBM. Tecnologia e organização social das práticas de saúde: características tecnológicas do processo de trabalho na rede estadual de centros de saúde de São Paulo. São Paulo: HUCITEC; 1994.

(17) Testa M. Pensar em saúde. Porto Alegre: Artes Médicas; 1992.

(18) Ferraz CA. As dimensões do cuidado em enfermagem: enfoque organizacional. Acta Paul Enferm 2000; 13(n. esp Pt 1):91-7.

(19) Marques GQ, Lima MADS. As tecnologias leves como orientadoras dos processos de trabalho em serviços de saúde. Rev Gaúcha Enferm 2004; 25(1):17-25.

(20) Fracolli LA, Maeda ST. A gerência nos serviços públicos de saúde: um relato de experiência. Rev Esc Enferm USP 2000; 34(2):213-7. 
O arquivo disponível sofreu correções conforme ERRATA publicada no Volume 40 Número 2 da revista. 